\title{
A Novel Fiber-Optic Tapered Long-Period Grating Sensor for Pressure Monitoring
}

\author{
Wojtek J. Bock, Fellow, IEEE, Jiahua Chen, Predrag Mikulic, and Tinko Eftimov
}

\begin{abstract}
The method and the required installations for fabricating tapered long-period fiber gratings can be simpler than that of standard fiber Bragg gratings, and the fabrication process is faster. To our knowledge, the tapered long-period fiber grating pressure sensor is presented here for the first time. In this paper, the fabrication method for tapered long-period fiber gratings, the sensing principle, the sensor structure, the measurement setup, and the preliminary results are presented and discussed. The pressure sensitivity of the sensor is as high as $5.1 \mathrm{pm} / \mathrm{bar}$.
\end{abstract}

Index Terms-Fiber-optic sensors, long-period fiber gratings, pressure calibration and measurement, step index fiber, tapered long-period gratings (TLPGs), temperature.

\section{INTRODUCTION}

$\mathbf{F}$ IBER-OPTIC Bragg gratings (FBGs) and a plethora of associated devices and systems are today finding wide use in areas covering telecommunications and sensor technology, especially for applications in structural and environmental monitoring. The operation of such devices is usually based on the well-known effect of UV-laser-induced periodic changes of the refractive index profile in the optical core of the fiber, which assures selective transmission and reflection of light. This effect has been exploited for many years, and the technology based on it is now approaching its limits in terms of physical performance.

Recently, a new class of Bragg gratings has emerged [1] in which the period $\Lambda$ of the waveguide structure modulation corresponds to a phase-matching condition between the fundamental mode of the optical core and a forward-propagating cladding mode. This period, which can go up to $1 \mathrm{~mm}$, is several orders of magnitude greater than in the case of classical FBGs; the corresponding devices are called long-period Bragg gratings (LPGs). These devices can have custom-designed sensitivities that may be much greater than in classical short-period FBGs to the specific parameters of interest. For this reason, their potential for the development of novel sensing devices should further be investigated. However, the UV-based technology to manufacture them is still very complex, time consuming, and expensive, which is impeding their wider implementation.

Manuscript received June 30, 2006; revised March 20, 2007. This work was supported in part by the Natural Sciences and Engineering Research Council of Canada, the Canada Research Chair Program, and the Canadian Foundation for Innovations.

The authors are with the Centre de recherche en photonique, Département d'informatique et d'ingénierie, Université du Québec en Outaouais, Gatineau, QC J8X 3X7, Canada (e-mail: wojtek.bock@uqo.ca).

Color versions of one or more of the figures in this paper are available online at http://ieeexplore.ieee.org.

Digital Object Identifier 10.1109/TIM.2007.899904
In this paper, we explore a concept involving the manufacturing of LPGs by using a computer-assisted-precision arc-discharge apparatus that we designed specifically for this purpose. This fabrication method is cost effective as there is no need for an expensive UV laser, complicated phase masks, or a vibration-isolated table. It is also time saving because the fiber alignment is straightforward. There is no need for complicated optical alignment and adjustment. The gratings manufactured this way have periodic melted and resolidified areas along their length, which produce periodic index and diameter variations. To date, few attempts using non-laser-based procedures have been reported in the literature [2], [3]. All of them lacked precision and repeatability and were characterized by the relatively low dynamic range (10-15 dB) of the manufactured devices. Recently, some LPGs written in a photonic crystal fiber [4] were tested to temperature, bending, and strain.

We also report in this paper the first successful application of such tapered long-period gratings (TLPGs) manufactured with the process described above for conducting direct measurements of hydrostatic pressure up to 450 bar. (Due to its widespread practical diffusion, the bar unit will be used in this paper. The conversion from bar to pascal, which is the International System of Units for pressure, is 1 bar equivalent to $100000 \mathrm{~Pa}$.) It is important to underline that classical FBGs have extremely low sensitivity to pressure equal to about $0.3 \mathrm{pm} / \mathrm{bar}$ [5] and therefore cannot be used directly for pressure measurements. The only way this task could be performed with FBGs is through detecting external strain in internally pressurized tubes or vessels that have FBGs glued to their outer surfaces, which is associated with an additional loss of accuracy.

\section{INSTRUMENTATION SETUP}

The objective of this paper is to design, manufacture, and test the first pressure sensors ever developed based on tapered LPGs and applied directly for hydrostatic pressure measurements up to 450 bar.

\section{A. LPG Fabrication}

The LPGs were manufactured using our computer-assistedprecision arc-discharge apparatus on the basis of a standard SMF-28 optical fiber designed for single-mode operation at $1500-\mathrm{nm}$ wavelength. The grating pitch $\Lambda$ varied from 0.6 to $0.8 \mathrm{~mm}$ and was determined by the moving step of the translation stage that was controlled by a computer. The grating length $L$ was in the range of $10-40 \mathrm{~mm}$, and the arc discharge 


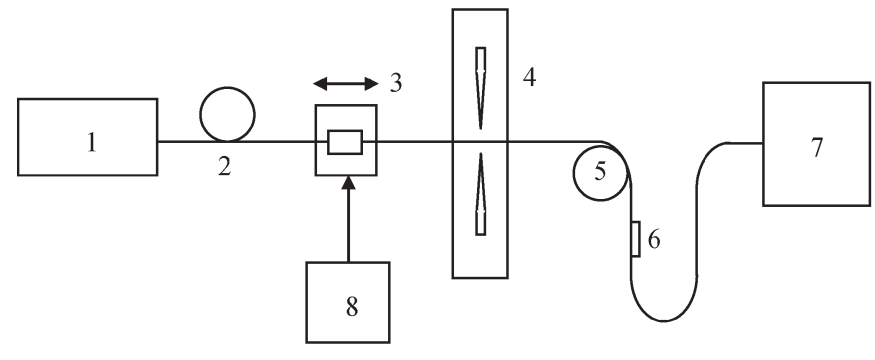

Fig. 1. Computer-assisted arc-discharge apparatus for manufacturing optical LPG sensors. (1) Broadband light source. (2) Single-mode optical fiber. (3) Motorized precision translation stage. (4) Arc-generating electrodes. (5) Pulley. (6) Weight. (7) OSA. (8) Computer.

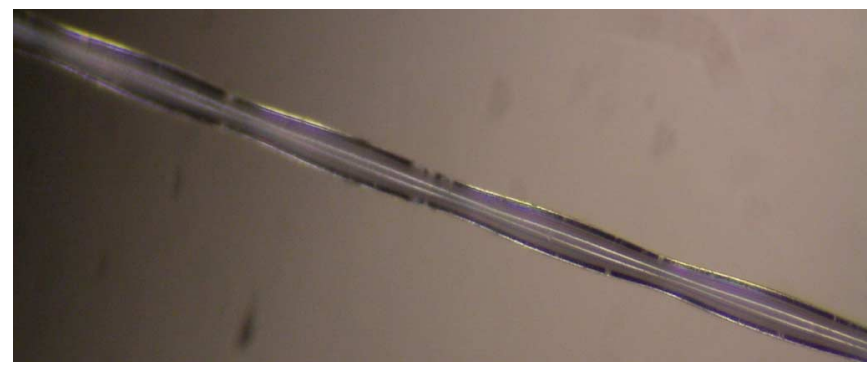

Fig. 2. Outer shape of a tapered LPG.

time $\tau$ was established at $400 \mathrm{~ms}$. During the process of LPG fabrication, an optical spectrum analyzer (OSA) was used to monitor their output characteristics. The setup of this apparatus is schematically presented in Fig. 1. The pulley and the weight shown in the figure were used to straighten the optical fiber.

The spectral properties of LPGs are determined by the mode coupling between the guided $\mathrm{LP}_{01}$ core mode and the $\mathrm{LP}_{0 i}$ cladding modes when a periodic perturbation of the refractive index of a pitch $\Lambda$ is introduced along the fiber. As the cladding modes are strongly attenuated, coupling of the guided mode to a cladding mode leads to the minima in the transmission spectra at wavelengths determined by

$$
\begin{aligned}
\lambda_{i} & =\delta n_{\mathrm{eff}, i} \cdot \Lambda \\
\delta n_{\mathrm{eff}, i} & =n_{\text {core }, \mathrm{eff}}^{01}-n_{\text {clad,eff }}^{0 i}
\end{aligned}
$$

where $n_{\text {core,eff }}^{01}$ is the effective refractive index of the guided core mode, $n_{\text {clad,eff }}^{0 i}$ is the effective refractive index of the $i$ th cladding mode, and $\delta n_{\mathrm{eff}, i}$ is their difference. This effect is physically the same as that in abrupt nonadiabatic fiber-optic tapers, which implies that if a periodic tapering is introduced along the fiber structure, a TLPG can be obtained.

The outer shape of a typical tapered LPG fabricated in our lab is shown in Fig. 2. The fiber diameter of the grating changes periodically from the maximum to the minimum. Usually, the minimum diameter varies from 115 to $120 \mu \mathrm{m}$.

A typical transmission spectrum obtained in our TLPGs made from Corning SMF-28 fiber is shown in Fig. 3. The figure clearly shows an excellent depth of the characteristic notch equal to $30 \mathrm{~dB}$ at $1574 \mathrm{~nm}$. In comparison, only about $15 \mathrm{~dB}$ is obtainable by other non-UV laser technologies.

\section{B. Sensor Structure}

Typical fiber Bragg gratings are not sensitive to touching and bending, but LPGs are. The output notch of an LPG is easily shifted or even deformed by changes of its inner stress and physical shape or of the surrounding material. In order to make an LPG pressure sensor capable of maintaining a good notch within the whole dynamic range of the applied pressure, it is necessary to keep the LPG straight at all times. There are two ways to achieve this: One is to always keep the grating at a certain constant axial tension. This is not difficult to achieve for fiber gratings used for stress sensing, since in this case the fiber grating is always pulled straight. For pressure sensing, however, a special housing depicted in Fig. 4(a) was developed for this purpose.

This housing was actually used for a pressure sensor developed in a transmission configuration. As can be seen from Fig. 4(a), the TLPG was kept straight in the small inner channel of a tube, and a fiber loop 4 was devised to keep the TLPG at almost constant axial tension at different temperatures and under different pressures. It is important not to allow the fiber loop 4 to swing because its position can greatly affect the shape and position of the output notch. The opening 8 was used for connecting the pressure sensor with a pressure calibration standard. The advantage of this kind of sensor is that the output light intensity is higher, but some disadvantages are unavoidable due to the existence of the fiber loop. First, the inner volume of the sensor housing is larger. This larger volume and the correspondingly larger pressure seal ring attached to the sensor housing lower the pressure limit that the sensor can withstand. Consequently, transmission-type sensors can only be used for lower pressure ranges. Second, the loop diameter changes with temperature, and this can create some torsion inside the fiber. Such torsion can affect the shape and position of the output notch in a detrimental way.

Fig. 4(b) shows another way to keep the grating straight in the reflection configuration and can entirely eliminate the possibility of any axial stress or torsion inside the fiber. The disadvantages of the reflection type are the need for a reflecting mirror at the distal end of the LPG and the requirement for a light source with higher output. There are, however, some obvious advantages: The sensor is compact, and at any temperature and under any pressure, the LPG is free of axial tension. This results in a deep output notch of an appropriate shape. The LPG pressure sensors presented in this paper were developed in reflection configuration.

\section{RESUlts}

The resonance wavelength of an LPG, such as the one depicted in Fig. 3, shifts under the influence of external parameters, and this is the foundation of the metrological applications of LPGs. Such a shift has already been determined in the case of temperature and strain; consequently, both features have been exploited in the development of novel fiber-optic sensors [4], [6].

However, the hydrostatic pressure sensitivity of tapered LPGs has never before been determined and is presented in this paper for the very first time. Fig. 5 shows an experimental setup 


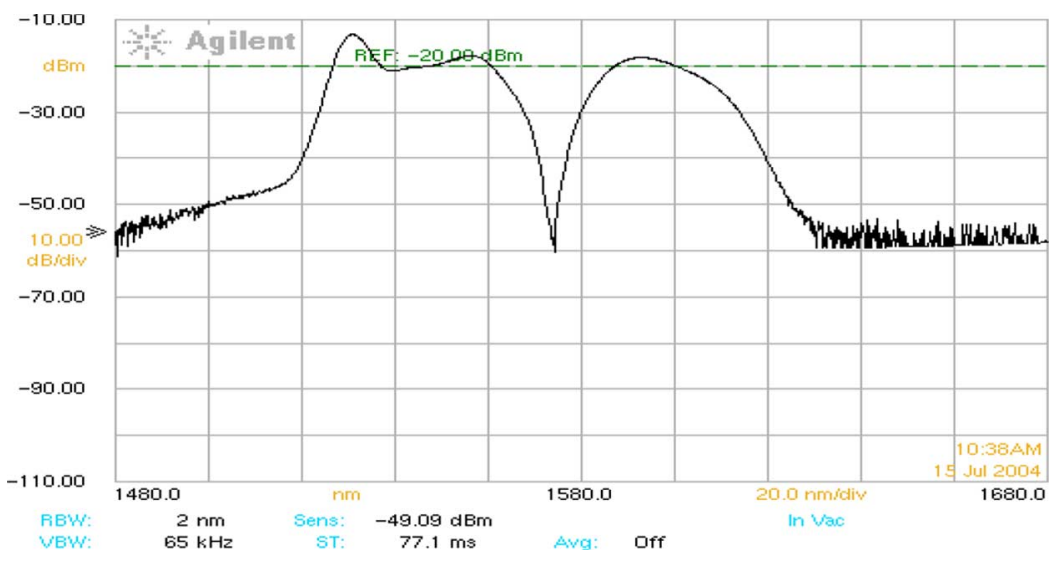

Fig. 3. Example of a transmission spectrum of a TLPG $(\Lambda=0.75 \mathrm{~mm}$, and $L=26 \mathrm{~mm})$.

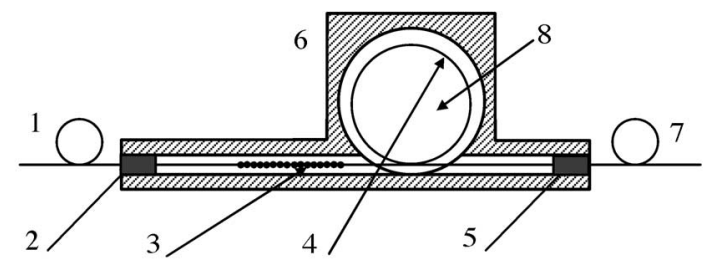

(a)

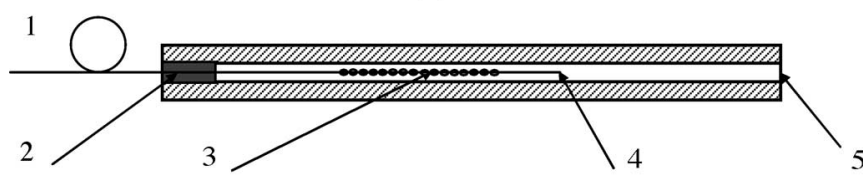

(b)

Fig. 4. Two possible configurations of TLPG pressure sensors. (a) Transmission configuration: ( 1 and 7) optical fiber; (2 and 5) glue; (3) TLPG; (4) optical fiber loop; (6) sensor housing; and (8) connecting opening. (b) Reflection configuration: (1) optical fiber; (2) glue; (3) TLPG; (4) reflecting mirror; and (5) connecting opening.

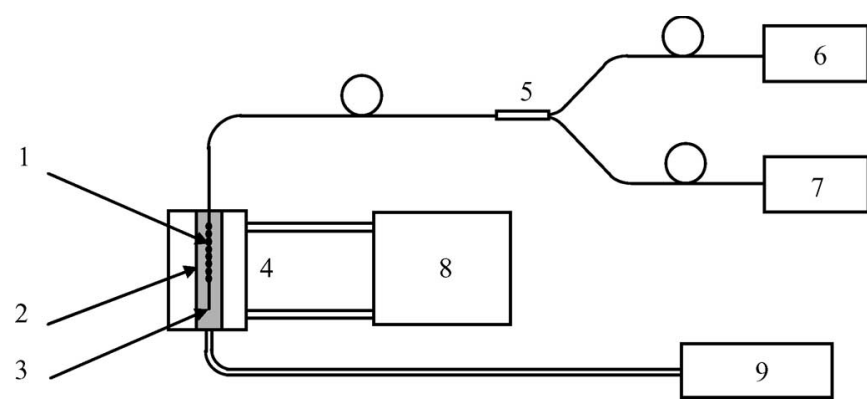

Fig. 5. Instrumentation setup to measure pressure and temperature sensitivities of TLPGs. (1) Tapered LPG. (2) Pressure sensor housing. (3) Reflecting mirror. (4) Oil container. (5) Optical fiber coupler. (6) Broadband optical source EXFO FPMD-5600 with spectral width from 1500 to $1600 \mathrm{~nm}$. (7) OSA Agilent $86142 \mathrm{~B}$ with a minimum resolution of $0.001 \mathrm{~nm}$. (8) Temperature circulator Julabo F32. (9) Deadweight pressure standard DWT-35 with accuracy of $0.01 \%$ fs.

used to determine the pressure and temperature sensitivities of these LPGs. The same setup can also be used to calibrate such fiber-optic sensors in reflection configuration against the primary pressure standard.

The temperature and hydrostatic pressure sensitivities of a variety of TLPGs were measured using the setup presented in Fig. 5. This instrumentation system is based on a reflective approach in which a TLPG is powered by a broadband optical

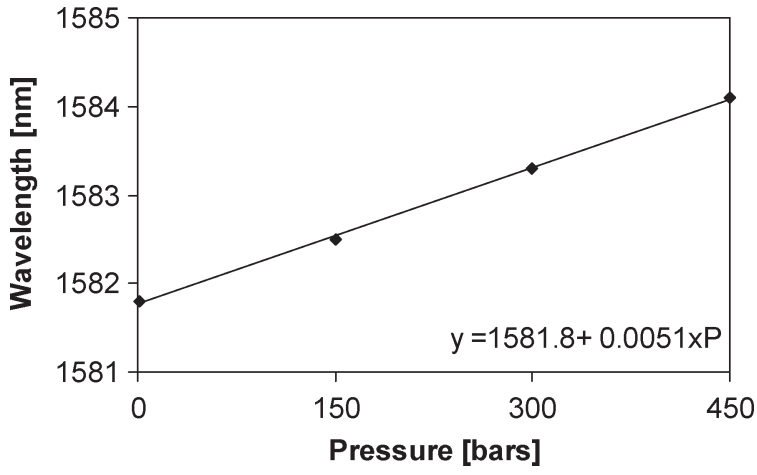

Fig. 6. Example of the output signal of a TLPG sensor as a function of hydrostatic pressure.

source via a 50-50 fiber-optic coupler, and the output spectrum is monitored by an OSA via the same coupling device. The reflecting mirror was made by depositing aluminum on the distal end of the grating prior to the experiment by means of a sputtering process conducted using our multifunction Lesker vacuum deposition cluster. Although our chosen instrumentation approach requires the creation of a high-quality mirror on the fiber tip, the advantage is obvious in that just one lead-in and one lead-out fiber are used, which results in a much smaller and compact sensing probe.

A typical sensor output signal is a linear function of pressure in the case of our TLPGs. For a TLPG with pitch $\Lambda=0.75 \mathrm{~mm}$ and length $L=26 \mathrm{~mm}$, the pressure characteristic of the sensor output signal for a range of pressures up to 450 bar is shown in Fig. 6. The output characteristic is similar to that of a typical Bragg grating strain sensor [6].

Practically, any other range can be covered as well by an appropriate sensor design according to the specific requirements. The developed LPG device displays significant potential as a highly sensitive and cost-effective pressure sensor with pressure sensitivity of about $5.1 \mathrm{pm} / \mathrm{bar}$, which is more than an order of magnitude more sensitive to pressure changes than a standard FBG [4]. At the same time, the temperature drift of the resonance wavelength in our gratings is still smaller than that reported for other gratings [6].

The thermal characteristic of the pressure sensor was measured from $5{ }^{\circ} \mathrm{C}$ to $65{ }^{\circ} \mathrm{C}$, and the results are given in Fig. 7 . During the measurement, the sensor was left at an atmospheric 


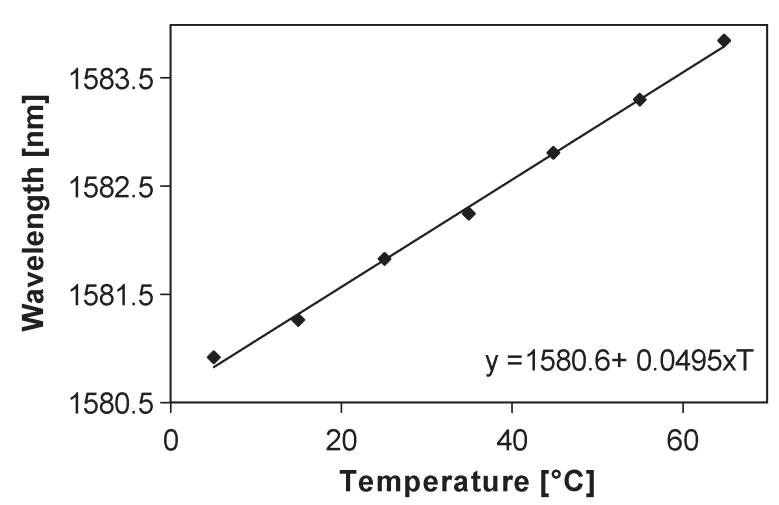

Fig. 7. Temperature effect on the output of a TLPG pressure sensor.

pressure environment. The temperature coefficient is about $50 \mathrm{pm} /{ }^{\circ} \mathrm{C}$. The temperature coefficients for the long-period fiber gratings and for the standard fiber Bragg gratings made from $\mathrm{B} / \mathrm{Ge}$ codoped fibers have been compared, and they are higher for the LPGs [7]. The reason for this is that the coupling between the core mode and the cladding mode is sensitive to the temperature property of the fiber material used for the LPGs. Our recent research on long-period fiber gratings has revealed that using a suitable kind of photonic crystal fiber, for instance, a single-mode photonic crystal fiber to replace Corning single-mode fiber SMF-28 could greatly reduce the temperature coefficient of an LPG pressure sensor.

\section{Sensing PRinciples}

The behavior of the tapered LPG with changes of pressure and temperature can be qualitatively explained using the fact that the propagation constant $\beta$ of a mode in a step index fiber can be written as [8] that in (3), shown at the bottom of the page, or as

$$
\beta^{2} \leq k^{2} \cdot I \cdot A
$$

with

$$
\begin{aligned}
I & =\int_{0}^{\infty} n^{2}(r) R^{2}(r) r d r \\
k & =\frac{2 \pi}{\lambda_{0}} \\
A & =\left(\int_{0}^{\infty} R^{2}(r) r d r\right)^{-1}
\end{aligned}
$$

where $n(r)$ is the radial distribution of the refractive index, $R(r)$ is the radial mode field function, $r$ is a radial variable, $m$ is an integer, and $\lambda_{0}$ is light wavelength in free space. Because core mode fields are mainly confined in the fiber core, thus, from (5), we get

$$
I_{\text {core }}=n_{\text {core }}^{2} \int_{0}^{a} R_{\text {core }}^{2}(r) r d r
$$

where $n_{\text {core }}$ is the refractive index of the core, which is a constant, and $a$ is the radius of the core. Cladding modes mainly exist in both core and cladding regions, and therefore,

$$
I_{\text {clad }}=\int_{0}^{d} n^{2}(r) R_{\text {clad }}^{2}(r) r d r
$$

where $d$ is the radius of cladding. By using (6), (7), and

$$
\beta=k \cdot n_{\mathrm{eff}}
$$

we can find from (5) the relations between the effective refractive index and the mode fields, i.e.,

$$
\begin{aligned}
& n_{\text {core }, \text { eff }}^{2} \sim I_{\text {core }} \cdot A \\
& n_{\text {clad }, \text { eff }}^{2} \sim I_{\text {clad }} \cdot A .
\end{aligned}
$$

The pressure along the radial direction will make the cladding diameter smaller but will not greatly affect the size of the core diameter because the core diameter is much smaller than that of the cladding. According to (7), under the action of this kind of pressure, $I_{\text {clad }}$ will become smaller, and hence, from (10), $n_{\text {clad,eff }}$ will diminish. According to (1), the radial pressure will make the coupling wavelength of the cladding mode longer, and this situation is reflected in Fig. 6.

As for the temperature effect, there are two things to consider: the increase of temperature will increase the grating pitch and the core radius. For a step index fiber, the normalized frequency $V$ is defined as

$$
V=k a \sqrt{n_{\text {core }}^{2}-n_{\text {clad }}^{2}}
$$

and the curve of the effective refractive index versus normalized frequency shows $n_{\text {eff }}$ monotonously increasing with $V$ [9]. From (8) and (11), it is easy to draw the conclusion that the propagation constant $\beta$ monotonously increases with the core radius $a$. Therefore, the increase of core radius will increase the propagation constant of the core mode and, hence, will increase the effective refractive index of the core. With these two reasons, it will be easy to use (1) to explain why the coupling wavelength of the cladding mode increases with increasing temperature, as seen in Fig. 7.

$$
\beta^{2}=\frac{\int_{0}^{\infty} k^{2} n^{2}(r) R^{2}(r) r d r-\int_{0}^{\infty}\left\{[d R(r) / d r]^{2}+\left(m^{2} / r^{2}\right) R^{2}(r)\right\} r d r}{\int_{0}^{\infty} R^{2}(r) r d r}
$$




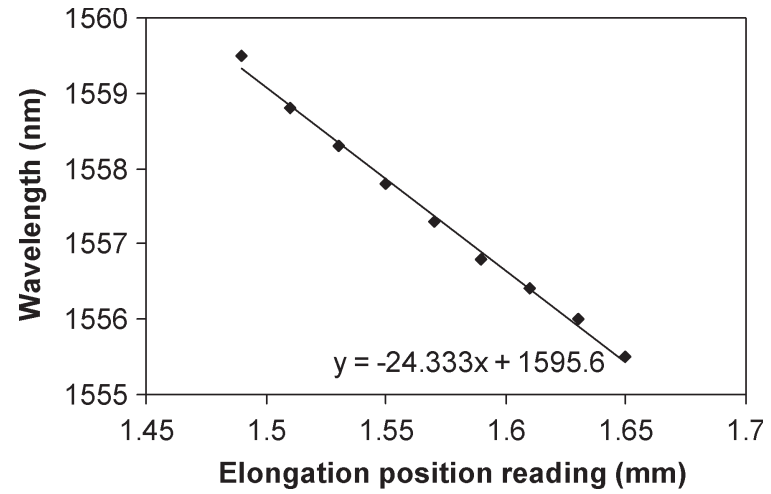

Fig. 8. Example of elongation effect on the output of a TLPG.

Our experiments have shown that in (1), the effective refractive indices play a more important role than the grating pitch. This is confirmed in Fig. 8, which shows notch movement under the action of elongation of a TLPG with the grating length of about $23 \mathrm{~mm}$.

When the grating is forced to elongate, the core diameter becomes smaller, and the pitch becomes longer too. The former decreases the propagation constant of the core mode and in consequence decreases the effective core refractive index of that mode, but the latter increases the pitch of the grating. The total effect is determined by the dominant factor. In this case, in (1), it is the core effective refractive index that plays a major role, and its decrease makes the grating output notch move toward the shorter wavelength, as reflected in Fig. 8.

The sensitivities of the resonant wavelength $\lambda_{i}$ to temperature $T$, strain $\varepsilon$, and pressure $p$ are

$$
\begin{aligned}
S_{T}^{i} & =\frac{d \lambda_{i}}{d T} \\
S_{\varepsilon}^{i} & =\frac{d \lambda_{i}}{d \varepsilon} \\
S_{p}^{i} & =\frac{d \lambda_{i}}{d p} .
\end{aligned}
$$

By using (1) and relation

$$
d \Lambda / \Lambda=d L / L
$$

(12)-(14) can be written as

$$
\begin{aligned}
S_{T}^{i} & =\Lambda \frac{d\left(\delta n_{\mathrm{eff}, i}\right)}{d T}+\lambda_{i} \frac{1}{L} \frac{d L}{d T} \\
S_{\varepsilon}^{i} & =\Lambda \frac{d\left(\delta n_{\mathrm{eff}, i}\right)}{d \varepsilon}+\lambda_{i} \\
S_{p}^{i} & =\Lambda \frac{d\left(\delta n_{\mathrm{eff}, i}\right)}{d p}+\lambda_{i} \frac{1}{L} \frac{d L}{d p}
\end{aligned}
$$

where $L$ is the grating length. It should be noticed that under the action of radial pressure, the second term on the right side of (17) does not play an important role because in this case $d L / d p$ can be neglected. In the above expressions, the first and second terms stand for the material and waveguide effects. The resultant resonant wavelength shift of the $i$ th peak will then be

$$
\Delta \lambda_{i}=S_{T}^{i} \Delta T+S_{\varepsilon}^{i} \Delta \varepsilon+S_{p}^{i} \Delta p
$$

where $\Delta T, \Delta \varepsilon$, and $\Delta p$ are increments of temperature, stress, and pressure, respectively.

In our case, coupling of the $\mathrm{LP}_{01}$ core mode to only one $\mathrm{LP}_{0 i}$ cladding mode is considered; hence, we omit the upper $i$ indices.

The length of the grating used for obtaining Figs. 6 and 7 is about $26 \mathrm{~mm}$, and from the plots, we find that $S_{T}^{i}=49.5 \times$ $10^{-3} \mathrm{~nm} /{ }^{\circ} \mathrm{C}$, and $S_{p}^{i}=5.1 \times 10^{-3} \mathrm{~nm} / \mathrm{bar}$. The length of the grating used for obtaining Fig. 8 is about $23 \mathrm{~mm}$. With the parameters shown in Fig. 8 and the assumption of uniform extension, the strain sensitivity is calculated as $S_{\varepsilon}^{i}=-0.6 \times$ $10^{-3} \mathrm{~nm} / \mu \varepsilon$.

These results are meaningful for the development of multiparameter all-fiber sensors.

\section{CONClusion}

We have developed a simple technology to manufacture tapered long-period fiber-optic gratings using a computercontrolled-precision arc-discharge apparatus that we assembled. The output notch depth of our TLPG reaches up to $30 \mathrm{~dB}$, which significantly exceeds that of the standard UV-written LPGs. We show for the first time that the pressure sensitivity of tapered LPGs is an order of magnitude greater compared to standard FBGs, and we report the construction of a compact reflective type of a fiber-optic pressure sensor based on TLPGs.

\section{REFERENCES}

[1] V. Bhatia, "Application of long-period gratings to single and multiparameter sensing," Opt. Express, vol. 4, no. 11, pp. 457-466, May 1999.

[2] K. Morishita et al., "Fabrication and resonance wavelengths of long-period gratings written in a pure silica photonic crystal fiber by the glass structure change," J. Lightw. Technol., vol. 22, no. 2, pp. 625-630, Feb. 2004.

[3] G. Humbert et al., "Long period grating filters fabricated with electric arc in dual concentric core fibers," Opt. Commun., vol. 225, no. 1-3, pp. 47-53, Sep. 2003.

[4] H. Dobb, K. Kalli, and D. J. Webb, "Measured sensitivity of arc-induced long-period grating sensors in photonic crystal fibre," Opt. Commun., vol. 260, pp. 184-191, 2006.

[5] M. G. Xu et al., , "Optical in-fiber grating high pressure sensing," Electron. Lett., vol. 29, no. 4, pp. 398-399, Feb. 1993.

[6] K. J. Han et al., "Simultaneous measurement of strain and temperature incorporating a long-period fiber grating inscribed on a polarization-maintaining fiber," IEEE Photon. Technol. Lett., vol. 16, no. 9, pp. 2114-2116, Sep. 2004.

[7] T. S. Francis and S. Yin, Fiber Optic Sensors. New York: Marcel Dekker, 2002.

[8] T. Okoshi, Optical Fibers. New York: Academic, 1982.

[9] W. van Etten and J. van der Plaats, Fundamentals of Optical Fiber Communications. Englewood Cliffs, NJ: Prentice-Hall, 1991.

Wojtek J. Bock (M'85-SM'90-F'03), photograph and biography not available at the time of publication.

Jiahua Chen, photograph and biography not available at the time of publication.

Predrag Mikulic, photograph and biography not available at the time of publication.

Tinko Eftimov, photograph and biography not available at the time of publication. 